\title{
EDITORIAL
}

\section{IMMUNOTHERAPY FOR ADVANCED COLON CANCER CURRENT STATUS AND FUTURE PROSPECTS}

\author{
Yasuhisa Koyanagi and Nobuaki Sakamoto
}

\begin{abstract}
Department of Surgery, Tokyo Medical University, 6-7-1 Nishi-Shinjuku Shinjuku-ku, Tokyo 160-0023, Japan
\end{abstract}

Key Words: Colon cancer, immunotherapy, interleukin-2, lentinan, Th1/Th2, Tc1/Tc2. Subjects: Patients.

Abbreviations: $\mathrm{CTL}=$ Cytotoxic lymphocyte, $\mathrm{IL}-2=$ interleukin $2, \mathrm{PSK}=$ polysaccharide $\mathrm{K}$

In recent years, the prognosis of colon cancer has shown marked improvement because of advances in diagnosis and treatment, and the 5-year survival rate exceeds $80 \%$ in patients undergoing curative resection (Japanese Research Society for Cancer of Colon and Rectum 1990). However, recurrence or metastasis still occurs in about $20 \%$ of the patients, and the outcome is not favorable. Among the various cancers of the gastrointestinal tract, colon cancer generally shows a poor response to chemotherapy, immunotherapy, and immunochemotherapy. No effective treatment has yet been established for inoperable patients or those with recurrent cancer. At present, research is being undertaken on various aspects of this problem, but it is essential to reconsider cancer treatment not only from the standpoint of experimental data, but also from the standpoint of treating the whole patient. Surgical techniques have largely been established, and this problem must be solved by developing better ancillary therapies. Chemotherapy should be reexamined not only with respect to efficacy, but also with respect to adverse reactions. From the $1980 \mathrm{~s}$, immunotherapy and immunochemotherapy have been widely used for gastrointestinal cancer, but the response cannot be called satisfactory. Antigen specific immunotherapy, such as cytotoxic T lymphocyte (CTL) therapy, has been performed by some institutions, which have reported a certain level of

Address request for reprints to: Dr. Yasuhisa Koyanagi, Department of Surgery, Tokyo Medical University, 6-7-1 Nishi-Shinjuku Shinjuku-ku, Tokyo 160-0023, Japan. Tel: 813-3342-6111, Fax: 81-3-3340-4575. 
efficacy. Cancer is immunologically complex and colon cancer has different biological characteristics in different patients. It is assumed that researchers will study antigenspecific immunotherapy more widely in the future, but it will require some time before a standard method of treatment is established because of the complex procedures involved and the cost. There are few institutions which are presently undertaking either basic or clinical studies about CTL therapy on colon cancer, and almost all hospitals rely on conventional immunotherapeutic agents such as PSK, lentinan, and OK-432 that are covered by the Japanese health insurance scheme. Here we report on the treatment of advanced colon cancer, based on two clinical trials which we performed. The histological and clinical effects of lentinan administration before and after surgery were assessed in the Study I, while lentinan plus IL-2 therapy was studied with respect to the effect on the cytokine balance (Th1/Th2, Tc1 and Tc2) in the Study II. We also discuss the current significance and future prospects of conventional immunotherapy.

Study I: Administration of lentinan before and after surgery

In 1994 and 1995, we performed a randomized comparative study on the histological and clinical effects of lentinan, in an attempt to determine the optimal dose. The subjects were patients under 75 years of age with Dukes B or C advanced colon cancer which was "curable" on operation. Dukes A patients were excluded since they have a 5 -year survival rate of about $95 \%$ after surgery alone. The patients were divided into four groups: a control group, and $2 \mathrm{mg}, 4 \mathrm{mg}$, and $8 \mathrm{mg}$ lentinan groups. The lentinan groups were administered lentinan twice about one and two weeks before the operation and then were administered the specified dose from the first week postoperatively. All of the patients, including the controls, were administered $800 \mathrm{mg} /$ day of 5'-DFUR (Furtulon ${ }^{\circledR}$ ) orally from the fourth week postoperatively. Thirty-two patients were enrolled and 24 of them were subjected to analysis. There were six patients in the control group, five in the $2 \mathrm{mg}$ group, six in the $4 \mathrm{mg}$ group and seven in the $8 \mathrm{mg}$ group.

\section{a) Histological effects}

For details, refer to the literature. Lymphocytes infiltrating the tumor and lymphocytes present within cancer cells (Figure 1: emperipolesis, i.e., lymphocytes contact with cancer cells) showed a dose-dependent significant increase in the lentinan groups. Most of these cells were cytotoxic T lymphocytes (Koyanagi, Sakamoto 1994). 


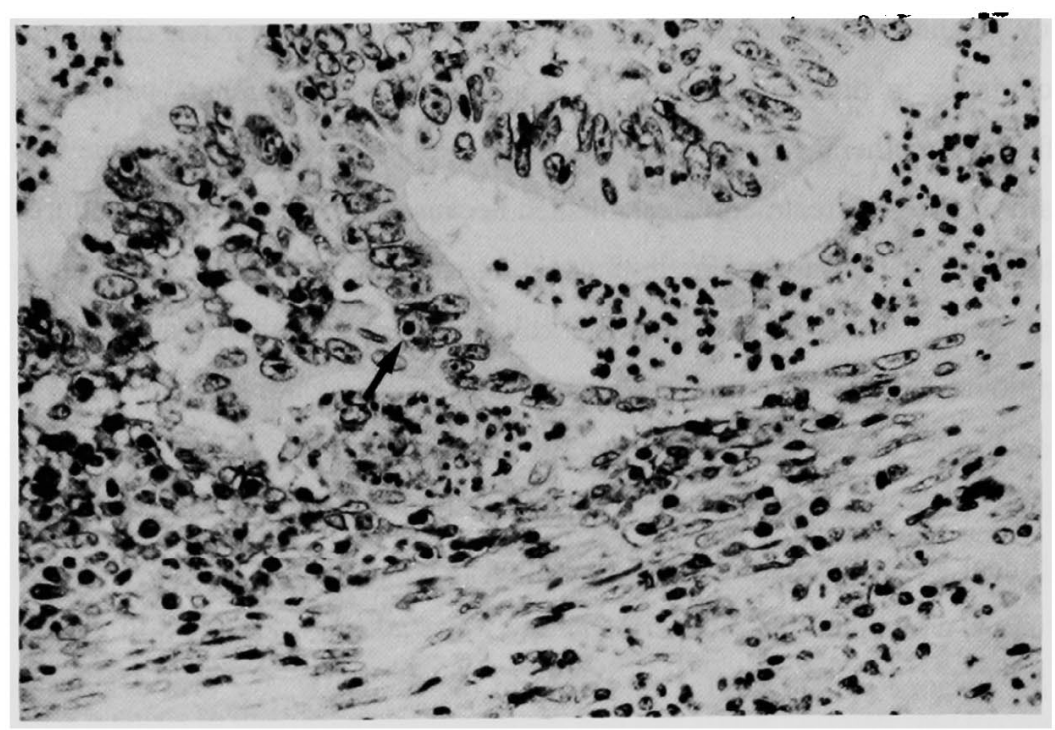

Figure 1.: Light microscopic detection of emperipoletic T lymphocytes (arrows) in the tumor $(\mathrm{X} 200)$

\section{b) Clinical outcome}

Figure 2 shows the cumulative recurrence-free survival rate. Only a few patients were analyzed and there were no statistically significant differences between any of the groups. However, no recurrence occurred in the $8 \mathrm{mg}$ group.

\section{c) Summary of Study I}

From the above results, it was concluded that the optimal dose of lentinan for advanced colon cancer patients is $8 \mathrm{mg} /$ week. (In a pre-clinical study, the incidence of adverse reactions tended to be high over $10 \mathrm{mg} /$ week, although they were not serious). Therefore, it is assumed that a higher dose than that currently used for gastric cancer (1 $\mathrm{mg}$ /week) is required to treat colon cancer. According to basic studies, a significantly better effect can be obtained with both preoperative and postoperative administrations of lentinan than with only preoperative or postoperative administration (Kimura, Koyanagi et al., 1996). It can be assumed that the increase of T-lymphocytes contact with cancer cells (emperipolesis) observed in this clinical trial is not only important for the localized effect on the tumor but also for prevention of recurrence, since lymphocytes contact with 


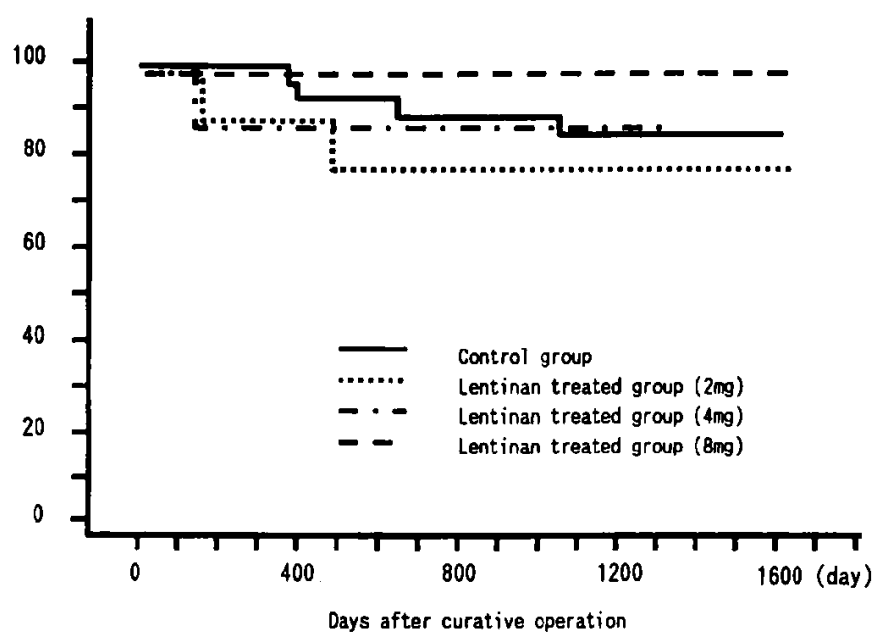

Figure 2.: Cumulative recurrence-free survival rate

cancer cells recognize tumor antigens and produce specific memory cells for such antigens (establishment of immune memory). These memory cells will effectively attack recurrent lesions, both locally and in distant organs when lentinan therapy is given postoperatively. Histopathological screening by preoperative administration assists in the prediction of responders and non-responders. Ohtani, Naito, Saito et al., (1999) also reported on the importance of microscopic emperipolesis when they detected "CD8positive lymphocytes within cancer nests". Lentinan increases the response to IL-2 by enhancing the expression of IL-2 receptors on lymphocytes, but it is not able to promote IL-2 production (Suzuki, Kikuchi et al., 1994; Hamuro, Takatsuki et al., 1994). Therefore, concomitant use of IL-2 is essential in advanced cancer patients with a reduced IL-2 production capacity.

Study II: Lentinan plus IL-2

Based on the above results, we performed a clinical trial in which both lentinan and IL-2 were administered to patients with advanced colon cancer from 1996 through 1998. The subjects fulfilled the same criteria as those in the first trial. The patients were below 75 years of age with Dukes B or C advanced colon cancer which could be treated with curative surgery. They were divided into three groups: a control group, a lentinan ( 8 $\mathrm{mg}$ ) plus low-dose IL-2 (100,000 JRU) group and a lentinan $(8 \mathrm{mg})$ plus high-dose IL-2 
VOL. 8, NOS. $1 \& 2$

2000
ANNALS OF

Cancer Research and Therapy
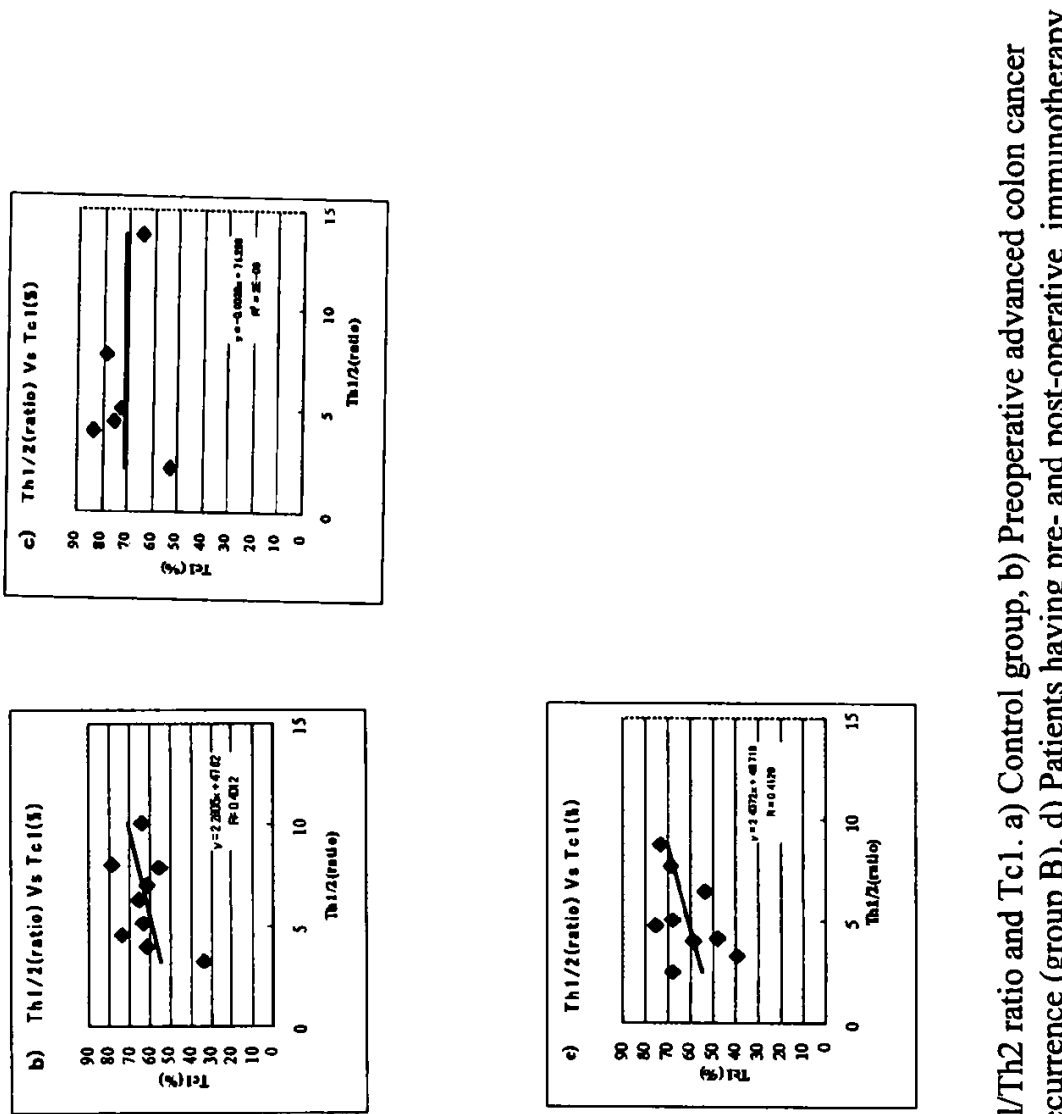

密

ํㅗㅇ

品拣

옵 옹

:

0 중

สิธ

่ิ

글 응

행

总号

롤

志䞤志

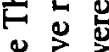

吾

娄

응

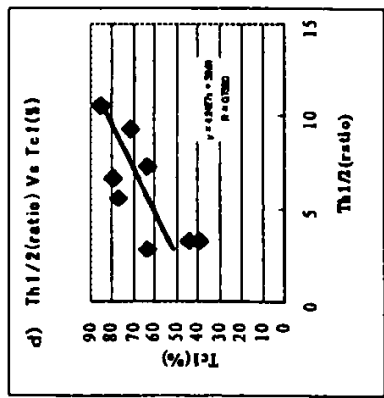

卷

도의

咲点

क

흥

若

ㅇํㄹ

.

蚂合。

ㅂ.

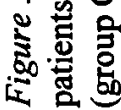


(400,000 JRU) group. The lentinan/IL-2-treated groups were administered lentinan / IL-2 twice about one and two weeks before the operation and then were treated from the first week postoperatively. The results of this clinical trial are currently being analyzed, but the histological findings were similar to those obtained with lentinan treated alone (unpublished data).

It has been reported that the $\mathrm{Th} 1 / \mathrm{Th} 2$ balance is impaired in cancer patients, $\mathrm{Th} 1$ activity become downregulation and $\mathrm{Th} 2$ cells tend to become predominant. In recent years, both in vitro and in vivo studies have shown that cytokines similar to Th1/Th2 cytokines are also secreted by both CD4 and CD8 cells, and they are known as Tc1 and Tc2 (Li, Sad et al., 1997; Mosmann, Sad, 1996; Mosmann, Li et al., 1997). However, no studies have clarified the role of $\mathrm{Tcl}$ and $\mathrm{Tc} 2$ in cancer patients. Therefore, we discuss the results obtained to date with respect to the cytokine balance together with the results obtained for patients excluded from the trial.

The subjects consisted of nine preoperative colon cancer patients (Group A), six patients with postoperative recurrence (Group B), eight patients having pre- and postoperative immunotherapy (lentinan + IL-2) (Group C), and eight patients that were untreated postoperatively (Group D). The control group consisted of ten healthy volunteers. Cytokines were analyzed by flow cytometry based on the method of Picker, Singh et al., (1995). IFN- $\gamma$ and IL-4 production by CD4 cells and CD8 cells was also measured.

a) IFN- $\gamma /$ IL-4 ratio in CD4 cells

The ratio was $7.65 \pm 3.63$ in the control group, $6.25 \pm 2.09$ in Group A, $6.19 \pm$ 3.77 in Group B, $5.82 \pm 2.67$ in Group C, and $5.28 \pm 2.06$ in Group D.

b) IFN- $\gamma / I L-4$ ratio in $C D 8$ cells

The ratio was $153.31 \pm 115.55$ in the control group, $64.14 \pm 62.96$ in Group $A$, $108.35 \pm 100.50$ in Group B, $55.18 \pm 37.53$ in Group C, and $30.89 \pm 23.90$ in Group D. c) Coefficient of correlation between the Th1/Th2 ratio and $T c 1$

In the control group, the correlation coefficient was 0.80 . It was 0.40 in Group A and 0.41 in Group D, while it was 0.73 in Group C. In Group B, there was no correlation and the Tcl level was high (Figure $3 \mathrm{a}-\mathrm{e}$ ). 


\section{d) Summary of Study II}

The above results indicate that not only the Th1/Th2 balance but also the $\mathrm{Tc} 1 / \mathrm{Tc} 2$ balance is impaired in cancer patients. When the immune function of cancer patients is studied, it is necessary to consider both the Th1/Th2 balance and also Tc1 activity. Immunotherapy which includes IL-2 may increase Tcl activity in association with improvement of the Th1/Th2 balance and lead to Tcl predominance. This is considered to be important with respect to the development of cachexia as well as the development of resistance to chemotherapy and immunotherapy in cancer patients, i.e., improving the Th1/Th2 balance is a key to improvement of the response to treatment.

\section{Future prospects}

Yoshino, Hazama et al., (1998) reported that IL-12 (p70), which plays an important role in the induction of the differentiation of $\mathrm{T}$ cells from Th0 to $\mathrm{Th} 1$, is increased by the administration of lentinan, while the Th2 cytokine IL-10 is reduced. They reported that it should be possible to modulate the Th1/Th2 balance by administration of OK-432 (a multiple cytokine inducer) after administration of lentinan (Yoshino, Hazama et al., 1998).

Hamuro and Murata (1998) classified macrophages as oxidative and reductive types. According to their report, IL-12 (which induces Thl cells) is produced by reductive macrophages; IL-12 in turn induces more reductive macrophages and converts oxidative macrophages into reductive macrophages. Lentinan also induces reductive macrophages, and the concomitant administration of lentinan with IL-2 potentiates the increase in reductive macrophages as well as potentiating the induction of IL-12 (Hamuro, Murata, 1998). These data provide important support for our clinical findings.

Many points remain to be clarified concerning the conventional immunotheraputic agents currently in clinical use, not only with respect to their actions but also the optimal dosage, administration method, and concomitant agents. In this article, we have concentrated on lentinan, but similar reports have appeared on other immunotheraputic agents. For example, OK-432 has been reported to induce both IL-12 and Th1 cells. It was recently reported that $\mathrm{OK}-432$ was an effective adjuvant in a clinical study of a vaccine produced by introducing the GM-CSF gene into mouse tumor 
cells, and there is a strong possibility that it will prove to be an important agent for concomitant therapy in the future (Abe, Wakimoto et al., 1995; Fujimoto, Duda et al., 1997).

PSK has also been shown experimentally to be effective against gastrointestinal cancer when administered orally. When a tumor cell line, colon 26, was transplanted subcutaneously or into the cecal wall in BALB/c mice and the local and systemic immune responses were compared, more potent systemic immunosuppression occurred and tumor growth was more rapid in mice with tumors transplanted into the cecum than in mice with subcutaneous transplantation. When the results of an oral administration of PSK were examined using this experimental system, the growth of colon 26 tumors in the cecal wall was inhibited dose-dependently by PSK. In addition, systemic immunosuppression was alleviated and TGF- $\beta$, which is considered to be the main circulating inhibitory factor, was significantly reduced when compared with the untreated group (Harada. Matsunaga et al., 1995, 1997).

The above reports indicate the new approaches being taken in basic research on conventional immunotheraputic agents. In the future, it is expected investigations from various aspects, including the analysis of responders and non-responders, may lead to new clinical applications for immunotherapy.

\section{References}

Abe, J., Wakimoto, H., Aoyagi, M., Hirakawa, K., Hamada, H. (1995) Cytokine-genemodified tumor vaccination intensified by a streptococal preparation OK-432. Cancer Immunol. Immunother. 41: 82-86.

Fujimoto, T., Duda, R.B., Szilvasi, A., Chen, X., Mai, M., O'Donnell, M.A. (1997) Streptococal preparation OK-432 is a potent inducer of IL-12 and a T helper cell 1 dominant state. J. Immunol. 158: 5619-5626.

Hamuro, J. and Murata, Y. (1998) Redox state of macrophages regulates the therapeutic effects of solid tumors. Hematol. Oncol. 36: 357-368, (Japanese).

Hamuro, J., Takatsuki, F., Suga, T., Kikuchi, T., Suzuki, M. (1994) Synergistic antimetastatic effects of lentinan and interleukin-2 with pre- and post-operative treatments. Jpn. J. Cancer. Res. 85: 1288-1297.

Harada, M., Matsunaga, K., Oguchi, Y., Ijima, H., Ito, O., Tamada, K., Kimura, G., Nomoto, K. (1995) The involvement of transforming growth factor $\beta$ in the impaired antitumor T-cell response at the gut associated lymphoid tissue (GALT). Cancer Res. 55: 6146-6151. 
Harada, M., Matsunaga, K., Oguchi, Y., Iijima, H., Tamada, K., Abe, K., Takenoyama, M., Ito, O., Kimura, G., Nomoto, K. (1995) Oral administration of PSK can improve anti-tumor CD4+ $\mathrm{T}$ cell response in gut associated lymphoid tissue (GALT) of specific-pathogene-free mice. Int. J. Cancer 70: 362-3721997.

Japanese Research Society for Cancer of Colon and Rectum. Multi-Institutional Registryof Large Bowel Cancer in Japan. Vol.16. Cases treated in 1990.

Kimura, K., Koyanagi, Y., Hirota, T., Saito, T., Kawaguchi, M., Serizawa, H., Kusama, M., Nakajima, A., Shimizu, T., Katoh, K., Sakamoto, N., Wada, T., Ogawa, N. (1996) Randomized comparative study of A-3021 in patients undergoing resection of colon cancer. Biotherapy. 10: 1533-1544 (In Japanese).

Koyanagi, Y. and Sakamoto, N. (1994) Emperipolesis at the light microscopic level - in the relationship between cytotoxic T lymphocytes and cancer cells. JJCS 19: 143143 (Japanese).

Li, L., Sad, S., Kagi, D., Mosmann, T.R. (1997) CD8 Tc1 and Tc2 Cells Secrete Distinct cytokine patterns in vitro and in vivo but induce similar inflammatory reactions. J. Immunol 158: 4152-4161.

Mosmann, T.R., Li, L., Sad, S. (1997) Functions of CD8 T-cell subsets secreting different cytokine patterns. Immunology 9: 87-92.

Mosmann, T.R. and Sad, S. (1996) The expanding universe of T-cell subsets: Th1, Th2 and more. Immunol. Today 138: 138-146.

Naito, Y., Saito, K., Shiiba, K., Ohuchi, A., Saigenji, K., Nagura, H., Ohtani, H. (1998) $\mathrm{CD} 8+\mathrm{T}$ cells infiltrated within cancer cell nests as a prognostic factor in human colorectal cancer. Cancer Res. 58: 3491-3494.

Picker, L.J., Singh, M.K., Zdraveski, Z., Treer, J.R., Waldrop, S.L., Bergstresser, P.R., Maino, V.C. (1995) Direct demonstration of cytokine synthesis heterogeneity among human memory/effector T cells by flow cytometory. Blood 85: 1408-1419.

Sakamoto, N., Koyanagi, Y., Nakajima, A., Kimura, K., Serizawa, H. (1995) Immunohistochemical analysis of advanced colon cancer after lentinan administration - Emperipolesis of tumor-infiltrating lymphocytes. Ann. Cancer Res. Ther. 4: 91-97.

Suzuki, M., Kikuchi, T., Takatsuki, F., Hamuro, J. (1994) Curative effects of combination therapy with lentinan and interleukin-2 against established murine tumors, and the role of CD8-positive T cells. Cancer Immunol. Immunother. 38: 1-8.

Yoshino, S., Hazama, S., Tabata, T., lizuka, N., Yamamoto, K., Hirasawa, K., Ogura, Y., Masaki, Y., Mori, N., Yoshimura, K., Oka, M. (1998) Immunotherapy based on Th1, Th2 theory. Biotherapy 12:1435-1440 (Japanese).

\section{COPYRIGHT @ $2000 \mathrm{BY}$}

PJD PUBLICATIONS LIMITED, P.O. BOX 966, WESTBURY, NY 11590

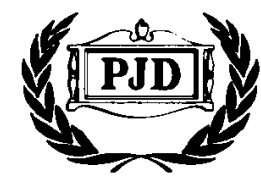

